\title{
Ancilla-assisted sequential approximation of nonlocal unitary operations
}

\author{
Hamed Saberi \\ Institute for Theoretical Physics, University of Regensburg, D-93040 Regensburg, Germany, \\ Physics Department, Arnold Sommerfeld Center for Theoretical Physics, and Center for NanoScience, \\ Ludwig-Maximilians-Universität, Theresienstrasse 37, D-80333 Munich, Germany, and \\ Department of Physics, Shahid Beheshti University, General Campus, Evin, Tehran 19839, Iran
}

(Received 18 August 2011; published 16 September 2011)

\begin{abstract}
We consider the recently proposed "no-go" theorem of Lamata et al. [Phys. Rev. Lett. 101, 180506 (2008)] on the impossibility of sequential implementation of global unitary operations with the aid of an itinerant ancillary system and view the claim within the language of Kraus representation. By virtue of an extremely useful tool for analyzing entanglement properties of quantum operations, namely, operator-Schmidt decomposition, we provide alternative proof to the no-go theorem and also study the role of initial correlations between the qubits and ancilla in sequential preparation of unitary entanglers. Despite the negative response from the no-go theorem, we demonstrate explicitly how the matrix-product operator (MPO) formalism provides a flexible structure to develop protocols for sequential implementation of such entanglers with an optimal fidelity. The proposed numerical technique, which we call variational matrix-product operator (VMPO), offers a computationally efficient tool for characterizing the "globalness" and entangling capabilities of nonlocal unitary operations.
\end{abstract}

DOI: 10.1103/PhysRevA.84.032323

PACS number(s): 03.67.Lx, 03.67.Bg, 02.70.-c, 71.27.+a

\section{INTRODUCTION}

Engineering arbitrary global unitary operations entangling simultaneously multiple qubits is generically regarded to be a task of formidable difficulty, as it may require a complicated combination of exponentially many gates to approximate [1]. However, efficient implementation of such genuinely entangling multiqubit operations is of paramount importance in quantum computation. Recently, it has been wondered if the situation could be facilitated by devising an ancillaassisted decomposition of a multiqubit unitary operation into a sequence of ancilla-qubit unitary operations where each qubit is allowed to interact locally and only once with an itinerant ancillary system (e.g., a trapped multilevel atom coupled to a single mode of an optical cavity in the realm of cavity QED experiments [2-4]) and without measurements [5] (see Fig. 1). Equivalently, according to the suggested scenario, instead of entangling directly neighboring qubits $k$ and $k+1$, an ancilla $a$ intervenes to get entangled with qubit $k$, and the ancilla state is swapped afterward with that of qubit $k+1$. Such decomposition, however, immediately faced a resounding no and was proved to be impossible in general for genuinely entangling unitaries, with the controlled-NOT (CNOT) gate being the handiest counterexample [5]. A reductio ad absurdum proof strategy for such a "no-go" theorem implied the incompatibility of unitarity of the last constituent two-qubit operation and the deterministic nature of the protocol. More precisely, having introduced an ancillary system to convey entanglement throughout the register of qubits, we eventually wish the ancilla decouples from the qubits in the very last step. But it turns out that for arbitrary entangling unitary the ancilla always remains entangled with the qubit system, thereby spoiling the promised determinism.

In the present paper, we reconsider the issue of such a sequential quantum "factory" of unitary entanglers within the framework of Kraus representation [6] and operator-Schmidt decomposition $[7,8]$ and provide alternative proofs to the no-go theorem forbidding the realization of such a setup. It turns out that employing such tools provides a systematic and transparent approach to various aspects of the problem and reveals the insurmountable mathematical obstacles to realization of such a scenario. Moreover, despite this negative answer, we investigate the possibility of implementing a nonlocal unitary with a certain imperfect fidelity. In other words, we demonstrate it is always possible to satisfy both conditions of the sequentiality and unitarity of the ancilla-qubit operations at the same time, however, at the price of ending up with a sequentially implemented unitary whose action is not perfectly equivalent to the original global unitary but is rather closest to that in some sense. This will be realized by exploiting the tools from matrix-product operator (MPO) theory [9-11]. We also study the role of initial correlations between the ancilla and qubits upon sequential preparation of unitaries and the way they could ever affect the evolution of the joint ancilla-qubit system.

\section{SEQUENTIAL QUANTUM "FACTORY" OF UNITARY ENTANGLERS VIEWED IN THE LANGUAGE OF QUANTUM OPERATIONS}

Let us first illustrate explicitly what is exactly meant by claiming that no entangling unitary can be implemented in a sequential way: For this purpose, we suggest viewing the problem within the framework of quantum operations $[1,6]$ where the qubit chain is regarded as the principal system and the ancilla as the environment (see Fig. 2). For simplicity, let us consider for now the specific case of only two qubits, $N=2$. We shall later generalize our argument to the case of an arbitrary number of qubits $N$. Assuming that the qubits and ancilla start out in a product state $\rho_{12 a}=\rho_{12} \otimes \rho_{\mathrm{a}}$, the evolution of the state of qubits is given by

$$
\mathcal{E}\left(\rho_{12}\right)=\operatorname{Tr}_{\mathrm{a}}\left[U_{12 a}\left(\rho_{12} \otimes \rho_{\mathrm{a}}\right) U_{12 a}^{\dagger}\right]=\sum_{k} E_{k} \rho_{12} E_{k}^{\dagger},
$$


(a)

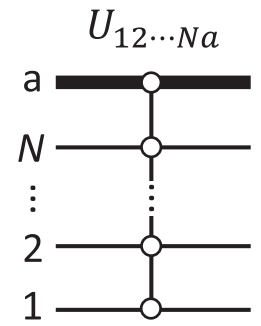

(b)

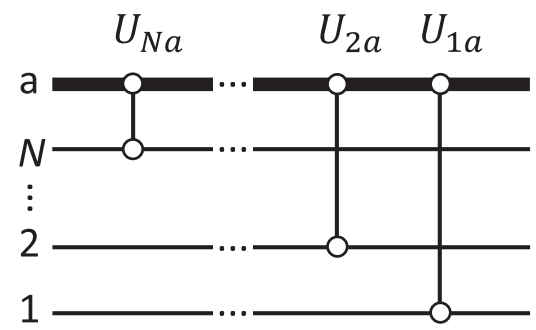

FIG. 1. Quantum circuit representation of (a) a generic nonlocal multiqubit unitary and (b) its ancilla-assisted sequential implementation. Each bipartite unitary $U_{k a}$ acts only on the Hilbert space of qubit $k$ and ancilla $a$ and leaves other qubits unchanged. To emphasize this fact, instead of the common circuit representation of an arbitrary bipartite unitary by a rectangular box, we have used vertical lines with open circles at each qubit they act upon. Ancilla states are shown with solid bold lines throughout.

where $E_{k} \equiv{ }_{a}\left\langle k\left|U_{12 a}\right| 0\right\rangle_{a}$ are Kraus operators in an operatorsum representation [1] of quantum operation $\mathcal{E}$ and $|k\rangle_{a}$ represent an orthonormal basis for the (finite-dimensional) state space of the ancilla; in particular, $|0\rangle_{\mathrm{a}}$ is assumed to be the initial state of the ancilla. We note that the aim is here to implement an entangling target two-qubit unitary $U_{12}$ with the aid of an ancilla $a$ whose action should be eventually factorized as $U_{12 a}=U_{12} \otimes \mathbb{1}_{a}$. Should the latter be the case, the Kraus operators are simply given by

$$
E_{k}={ }_{a}\left\langle k\left|U_{12} \otimes \mathbb{1}_{a}\right| 0\right\rangle_{a}=U_{12} \delta_{k 0} .
$$

The sequential preparation of the two-qubit unitary $U_{12}$, on the other hand, suggests Kraus operators of the form

$$
E_{k}={ }_{a}\left\langle k\left|\left(U_{2 a} \otimes \mathbb{1}_{1}\right)\left(\mathbb{1}_{2} \otimes U_{1 a}\right)\right| 0\right\rangle_{a} .
$$

Note that at step $k$ of the sequential implementation of a global $N$-body unitary operation $U_{12 \ldots N}$, the two-body unitary $U_{k a}$ entangles only the ancilla $a$ and qubit $k$ and leaves other qubits unchanged, the latter action having been denoted by a properly indexed identity operator $\mathbb{1}$ above. Now, if the entangling unitary $U_{12}$ could be implemented sequentially, $E_{1}$ in Eq. (3) should also vanish. Acting $E_{1}$ on some initial state of qubits 12 and performing Schmidt decomposition on the partition 1| $a$ of

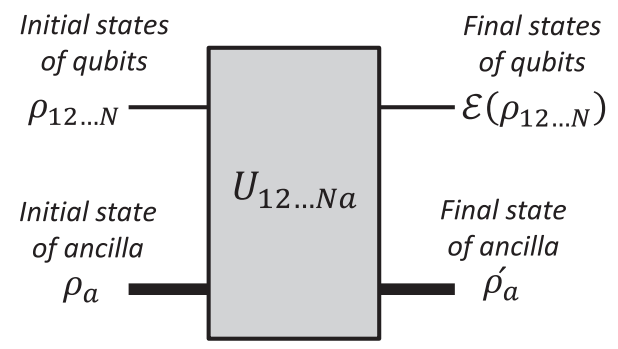

FIG. 2. Ancilla-assisted implementation of entanglers viewed as a quantum operation $\mathcal{E}\left(\rho_{12 \ldots N}\right)$. The joint ancilla-qubit system evolves under a unitary transformation $U_{12 \ldots N a}$.

the entangled state $U_{1 a}|00\rangle_{1 \mathrm{a}}$ yield

$$
\begin{aligned}
E_{1}|0 i\rangle_{12} & ={ }_{a}\left\langle 1\left|\left(U_{2 a} \otimes \mathbb{1}_{1}\right)\left(\mathbb{1}_{2} \otimes U_{1 a}\right)\right| 0 i 0\right\rangle_{12 a} \\
& =\sum_{j=0}^{\chi}{ }_{a}\left\langle 1\left|U_{2 a}\right| i j\right\rangle_{2 a}\left|\phi_{j}\right\rangle_{1}=0,
\end{aligned}
$$

with the Schmidt rank $\chi \geqslant 1$ [12], and by convention we have incorporated the Schmidt coefficients into the Schmidt vectors [13]. The orthogonality (and linear independence) of $\left|\phi_{\mathrm{j}}\right\rangle_{1}$ implies that the only way for $E_{1}$ to vanish for a sequentially prepared unitary would be that ${ }_{a}\left\langle 1\left|U_{2 a}\right| i j\right\rangle_{2 a}$ vanish for all $i$ and $j=0,1, \ldots$. The latter, however, causes two rows of $U_{2 a}$ and, consequently, its determinant to vanish, thereby violating the invertibility and, in turn, unitarity of $U_{2 a}$.

For sequential implementation of a global unitary one should satisfy two constraints of sequentiality and unitarity of the constituent ancilla-qubit operations. However, as illustrated above, these two cannot be fulfilled at the same time.

Note that the result above may be readily extended to an arbitrary number of qubits $N$ since it is always possible to map this general case to that of only two unitaries $U_{2 a} U_{1 a}$ by combining the first $n-1$ unitaries and the $n$th one, where $n$ is the smallest step at which the ancilla decouples from the qubit chain [5]. Therefore, for the sake of brevity, we shall henceforth only consider the paradigmatic case of $N=2$ and keep in mind that the results will accordingly be valid for an arbitrary number of qubits $N$ too.

We point out the "no-go" statement above holds even if we relax the assumption that the ancilla and qubits start out in a product state. To see this, we exploit the Fano form of the density matrix $[14,15]$ to follow the evolution of the joint ancilla-qubit system in the presence of initial correlations between the qubits as the open system [16] and the ancilla as the environment. Assuming the action of the ancilla can be factorized like $U_{12 a}=U_{12} \otimes \mathbb{1}_{a}$, the quantum operation then is given by [17]

$$
\mathcal{E}\left(\rho_{12}\right)=U_{12} \rho_{12} U_{12}^{\dagger}+\sum_{k}{ }_{a}\left\langle k\left|U_{12 a} \gamma_{i j}^{12 a} \sigma_{i}^{12} \otimes \tau_{j}^{a} U_{12 a}^{\dagger}\right| k\right\rangle_{a},
$$

where $\sigma_{i}$ and $\tau_{j}$ are the generators of $\operatorname{SU}(4)$ and $\operatorname{SU}(D)$, respectively, and $D$ is the dimension of the Hilbert space of the ancilla. Straightforward algebra then yields

$$
\mathcal{E}\left(\rho_{12}\right)=U_{12} \rho_{12} U_{12}^{\dagger}+\sum_{i, j} U_{12} \sigma_{i}^{12} U_{12}^{\dagger} \gamma_{i j}^{12 a} \operatorname{Tr}\left\{\tau_{j}^{a}\right\} .
$$

But generators of $\mathrm{SU}(D)$ are known to be traceless. So we conclude that for the case where the evolution of the whole ancilla-qubits is factorable with respect to the ancilla (or the ancilla decouples from the qubit chain), the initial correlations would not affect the final states of the qubits. Contrarily, for a nonfactorable sequentially implemented unitary, the correlation term in Eq. (6) does not necessarily vanish, and initial correlations may play a significant role in the evolution of the joint ancilla-qubit system and, in turn, in the final states of both qubits and the ancilla.

The main obstacle in sequential implementation of an entangling unitary, namely, the fact that the ancilla cannot be set to decouple from the qubit chain in the last step, may also be 
expressed in terms of the well-known problem of separability criteria in quantum information [18-21]: When we start from a 1-qubit biseparable state [22] of ancilla and qubits $\rho_{12 a}=\rho_{12} \otimes \rho_{a}$, straightforward algebra employing Kraus representation for both the ancilla and qubits implies that a factorable evolution $U_{12 a}=U_{12} \otimes U_{a}$ leaves such separability invariant, i.e., $\rho_{12 a}^{\prime}=\rho_{12}^{\prime} \otimes \rho_{a}^{\prime}$, where a prime indicates the final states. However, this ceases to be the case for the ancilla-assisted sequential evolution considered above.

\section{ALTERNATIVE PROOF TO THE NO-GO THEOREM BASED ON OPERATOR-SCHMIDT DECOMPOSITION}

In this section we provide an alternative proof to the no-go theorem by virtue of an extremely useful and elegant decomposition known in operator algebra as operator-Schmidt decomposition $[7,8]$, according to which the bipartite ancillaassisted unitaries may be decomposed as

$$
\begin{aligned}
& U_{2 a}=\sum_{i=1}^{\chi_{2}} A_{i}^{2} \otimes B_{i}^{a}, \\
& U_{1 a}=\sum_{i=1}^{\chi_{1}} A_{i}^{1} \otimes C_{i}^{a},
\end{aligned}
$$

with $\left\{A_{i}^{2}\right\},\left\{A_{i}^{1}\right\},\left\{B_{i}^{a}\right\}$, and $\left\{C_{i}^{a}\right\}$ being some orthogonal (but not necessarily normalized) operator bases defined on the Hilbert-Schmidt space of operators acting on qubits 2 and 1 and ancilla $a$, respectively. Of particular importance is $\chi_{2}$ $\left(\chi_{1}\right)$, called the Schmidt number of operator $U_{2 a}\left(U_{1 a}\right)$, defined as the number of nonzero coefficients in its operator-Schmidt decomposition. Applying the decomposition to each bipartite entangling unitary $U_{k a}$ yields

$$
\begin{aligned}
U_{12 a} & =\left(U_{2 a} \otimes \mathbb{1}_{1}\right)\left(\mathbb{1}_{2} \otimes U_{1 a}\right) \\
& =\left(\sum_{i} A_{i}^{2} \otimes B_{i}^{a} \otimes \mathbb{1}_{1}\right)\left(\mathbb{1}_{2} \otimes \sum_{j} A_{j}^{1} \otimes C_{j}^{a}\right) \\
& =\sum_{i, j} A_{j}^{1} \otimes A_{i}^{2} \otimes\left(B_{i}^{a} C_{j}^{a}\right) .
\end{aligned}
$$

Without loss of generality we may take $\chi_{2}=2$; the same argument would hold for an entangling the unitary of Schmidt number $\chi_{2}=4$ [23]. In order that the ancilla decouples unitarily from the qubit chain, it should be possible for it to be factorized like $B_{i}^{a} C_{j}^{a}=U_{a}$ for all $i$ and $j$ and with $U_{a}$ being some unitary operator on ancilla space. This in particular implies that $B_{1}^{a} C_{1}^{a}=B_{2}^{a} C_{1}^{a}$, eventually suggesting $U_{2 a}=\left(\sum_{i} A_{i}^{2}\right) \otimes B^{a}$ to be a nonentangling operation. The latter, however, is in contradiction to the original assumption that $U_{2 a}$ has a Schmidt number larger than 1 , and this readily completes the alternative proof.

\section{OPTIMAL IMPLEMENTATION OF NONLOCAL UNITARIES WITHIN THE FRAMEWORK OF MATRIX-PRODUCT OPERATORS}

Having accepted the impossibility of implementing a nonlocal unitary in a sequential way, we may wonder if we could rather try to approach a target unitary entangler within a sequential procedure, albeit in an approximate way and with an optimal fidelity. More precisely, the aim is then to find bipartite unitaries $U_{1 a}$ and $U_{2 a}$ that, when applied sequentially instead of the global unitary $U_{12 a}$, minimize the Frobenius norm distance [24]

$$
\begin{aligned}
\mathcal{C} & =\left\|U_{12 a}-\left(U_{2 a} \otimes \mathbb{1}_{1}\right)\left(\mathbb{1}_{2} \otimes U_{1 a}\right)\right\|_{F}^{2} \\
& =2 D-2 \operatorname{Re}\left\{\operatorname{Tr}\left[U_{12 a}^{\dagger}\left(U_{2 a} \otimes \mathbb{1}_{1}\right)\left(\mathbb{1}_{2} \otimes U_{1 a}\right)\right]\right\}
\end{aligned}
$$

Note here that we hereby require both sequentiality and unitarity conditions to be fulfilled without violating the abovementioned no-go theorem: In fact, these two plus the implicit condition that the sequentially implemented unitary should produce the same action of the global one (i.e., the identity $U_{12 a}|\Psi\rangle=\left(U_{2 a} \otimes \mathbb{1}_{1}\right)\left(\mathbb{1}_{2} \otimes U_{1 a}\right)|\Psi\rangle$ for arbitrary state $\left.|\Psi\rangle\right)$ do save the no-go theorem and make such a distance never vanish. Without such a condition, we could, of course, freely choose the sequential unitaries so that the ancilla decouples unitarily in the last step.

The constrained nonlinear optimization problem [25] associated with the cost function above may be solved efficiently by a variational search in the space of the MPOs arising from the sequential nature of the protocol. To see how operators of matrix-product form [9-11] arise naturally within such a prescription, we express each ancilla-qubit unitary in terms of an orthonormal basis

$$
U_{k a}=\sum_{i_{k}, j_{k}=0}^{1} \sum_{\alpha_{k}, \beta_{k}=1}^{D} U_{j_{k}, \beta_{k}}^{i_{k}, \alpha_{k}}\left|i_{k} \alpha_{k}\right\rangle\left\langle j_{k} \beta_{k}\right|,
$$

where Roman (Greek) letters denote qubit (ancilla) indices. The sequentially implemented unitary, as a result, reads

$$
\begin{aligned}
& \left(U_{2 a} \otimes \mathbb{1}_{1}\right)\left(\mathbb{1}_{2} \otimes U_{1 a}\right) \\
& \quad=\sum_{i_{2}, j_{2}} \sum_{\alpha_{2}, \beta_{2}} \sum_{i_{1}, j_{1}} \sum_{\alpha_{1}, \beta_{1}} U_{j_{2}, \beta_{2}}^{i_{2}, \alpha_{2}} U_{j_{1}, \beta_{1}}^{i_{1}, \alpha_{1}}\left|i_{2} \alpha_{2}\right\rangle\left\langle j_{2} \beta_{2}|\otimes| i_{1} \alpha_{1}\right\rangle\left\langle j_{1} \beta_{1}\right| \\
& =\sum_{i_{2}, j_{2}} \sum_{i_{1}, j_{1}} U_{[2]}^{i_{2}, j_{2}} U_{[1]}^{i_{1}, j_{1}}\left|i_{2}\right\rangle\left\langle j_{2}|\otimes| i_{1}\right\rangle\left\langle j_{1}\right|,
\end{aligned}
$$

where we have defined in the last line $\sum_{\alpha_{k} \beta_{k}} U_{j_{k}, \beta_{k}}^{i_{k}, \alpha_{k}}\left|\alpha_{k}\right\rangle\left\langle\beta_{k}\right| \equiv$ $U_{[k]}^{i_{k}, j_{k}}$ to cast the original expression into products of unitary operators. We may now easily recognize (11) as an MPO already encountered in the calculation of finite-temperature density matrices [26]. With this, the cost function (9) in the MPO representation reads

$$
\begin{aligned}
\mathcal{C}= & 2 D-2 \operatorname{Re}\left\{\operatorname { T r } \left(\sum_{j_{2}^{\prime}, j_{1}^{\prime}} \sum_{i_{2}, j_{2}} \sum_{i_{1}, j_{1}} U_{[21]}^{i_{2} i_{1}, j_{2}^{\prime} j_{1}^{\prime} \dagger} U_{[2]}^{i_{2}, j_{2}} U_{[1]}^{i_{1}, j_{1}}\right.\right. \\
& \left.\left.\otimes\left|j_{2}^{\prime} j_{1}^{\prime}\right\rangle\left\langle j_{2} j_{1}\right|\right)\right\},
\end{aligned}
$$

where we have expressed the target unitary too, in terms of an orthonormal basis, and have accordingly defined

$$
\sum_{\alpha^{\prime}, \beta^{\prime}} U_{\alpha^{\prime} \beta^{\prime}}^{i_{2}^{\prime} i_{1}^{\prime}, j_{2}^{\prime} j_{1}^{\prime}}\left|\alpha^{\prime}\right\rangle\left\langle\beta^{\prime}\right| \equiv U_{[21]}^{i_{2}^{\prime} i_{1}^{\prime}, j_{2}^{\prime} j_{1}^{\prime}}
$$

to have

$$
U_{12 a}=\sum_{i_{2}^{\prime}, j_{2}^{\prime}} \sum_{i_{1}^{\prime}, j_{1}^{\prime}} U_{[21]}^{i_{2}^{\prime} i_{1}^{\prime}, j_{2}^{\prime} j_{1}^{\prime}}\left|i_{2}^{\prime} i_{1}^{\prime}\right\rangle\left\langle j_{2}^{\prime} j_{1}^{\prime}\right| .
$$




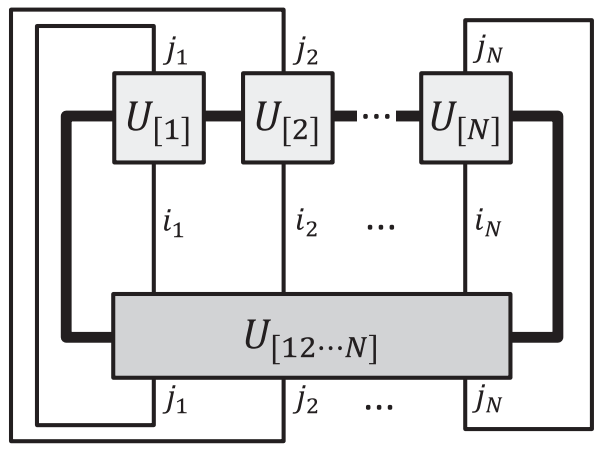

FIG. 3. Graphical representation of the overlap of MPOs used to calculate the contractions in the cost function equation (12). The square boxes represent $U_{[k a]}$ matrices of a sequentially prepared unitary, and the rectangular box represents the target global unitary. The links connecting the boxes represent indices that are being contracted (or summed over).

The calculation of the cumbersome summations on the righthand side of Eq. (12) finds a very simple form in MPO representation as illustrated in Fig. 3. The minimization problem can be done very efficiently in an iterative variational optimization technique (similar to those already used in the context of matrix-product states [4,27-30]) in which we fix all but one of the sequential unitaries, let's say $U_{[k]}$, and minimize the cost function equation (12) by varying over the matrix elements of $U_{[k]}$. In the next iteration, the neighboring unitary is optimized, and once all the unitaries so obtained have been optimized locally, we sweep back again and so on until convergence. In that sense, we suggest an extension of the previously developed method of variational matrix-product state (VMPS) [28-30] from states to operators and suggest calling it the variational matrix-product operator (VMPO) method.

In the case that the ancilla is a qubit, i.e., $D=2$, the two-qubit sequential unitaries of the variational MPO in Eq. (11) may be expanded in terms of the complete basis of Pauli matrices:

$$
U_{[k]}=\exp \left(-i \sum_{j_{1}, j_{2}=0}^{3} h_{j_{1}, j_{2}}^{[k]} \sigma_{j_{1}} \otimes \sigma_{j_{2}}\right),
$$

where $h_{j_{1}, j_{2}}^{[k]}$ are real-valued coefficients and $\sigma_{1}, \sigma_{2}$, and $\sigma_{3}$ are the usual Pauli sigma matrices, with $\sigma_{0} \equiv \mathbb{1}$ being the identity matrix. The minimization of the cost function equation (12) then amounts to finding the optimal coupling matrix $h^{[k]}$ at each step of the iterative optimization. The fidelity of the sequential implementation of the global unitary $U_{12 a}$ may be quantified then as $\mathcal{F}=1-\tilde{\mathcal{C}}$, where $\tilde{\mathcal{C}}$ denotes the normalized converged cost function $\mathcal{C}$ of Eq. (12) upon the very last sweep. The normalization is taken care of after dividing by the Frobenius norm of the involved operators.

We have applied the outlined procedure to some paradigmatic gates of quantum computing when the ancilla has the dimension $D=2$, as illustrated in Table I: For instance, the two locally equivalent gates CNOT and controlled- $Z$ (CZ) can be implemented sequentially with a fidelity of $70.71 \%$. The $29.29 \%$ error we associate with the existence of a "fidelity gap," a strict theoretical "red line" beyond which the global unitary cannot be implemented in a sequential fashion.
TABLE I. The values of the Frobenius fidelity for sequential implementation of various global gates with an ancilla of dimension $D=2$.

\begin{tabular}{ccccccc}
\hline \hline & \multicolumn{6}{c}{ Unitaries } \\
\cline { 2 - 7 } & CNOT & CZ & CPHASE & SWAP & Toffoli & Fredkin \\
\hline Fidelity & 0.7071 & 0.7071 & 0.9239 & 0.50 & 0.75 & 0.75 \\
\hline \hline
\end{tabular}

Similar optimization techniques may be exploited for higher ancilla dimension $D=4$, however, upon using the generators of SU(4) for the ancilla instead of Pauli matrices in Eq. (15) to see if it has any influence on the values of the gaps. We have performed such a simulation for the same gates as in Table I and have found that the values of the gaps remain unchanged for $D=4$ compared to that of $D=2$. Since $D=4$ is the maximum possible ancilla dimension for both $N=2$ and $N=3$ cases [31], we conclude that the reported values of the gaps are intrinsic to the gates, irrespective of the ancilla dimension.

We believe also that such intrinsic values of the gaps are closely related to the entanglement content of each unitary operation, the figure of merit for this purpose being the Schmidt strength [8] (proportional to the sum of nonzero Schmidt numbers of an operator) as a measure of operator entanglement [32-34]. Exemplifying values of Schmidt strengths are 0.5 and 0.75 for CNOT and SWAP, respectively. As expected, the larger the Schmidt strength of the gate is, the larger the corresponding fidelity gap is.

\section{CONCLUSIONS}

In conclusion, we have studied the problem of sequential implementation of entangling unitary operations within the various languages of Kraus representation, operator-Schmidt decomposition, and MPO representation. Exploiting such tools allowed us to revisit the previously proposed no-go theorem through a systematic and instructive scheme that reveals various mathematical aspects of the subject. The Kraus representation approach, in particular, enabled us to probe possible correlations between qubits and an ancilla after unitary evolution of the joint system and thereby to compare the ultimate action of sequentially prepared unitary to that of a global entangler. Employing MPO formalism, we also developed numerical techniques for an efficient realization of a sequential version of an entangling unitary operation with an optimal fidelity. As a realistic scenario, the recipe could instruct an experimentalist how to obtain optimal two-qubit operations that lead to a sequential version of the desired multiqubit entangler with the highest possible fidelity and without the need to perform any measurement.

We stress that these results and the proposed optimization protocols can be of wide potential applicability at the interface of condensed matter physics, quantum optics, and quantum information. In particular, they will be of direct relevance to any sequential physical setup like photonic qubits, superconducting qubits, or quantum dots. From a methodological point of view, the proposed numerical technique, the VMPO method, promises to be a computationally efficient tool for variational 
search into the Hilbert-Schmidt space of operators. We believe that the method may particularly be utilized for characterizing the entangling (and disentangling) properties of multiqubit unitary operations.

We point out, finally, that the no-go theorem does not refrain from implementing an entangling unitary if (instead of targeting the nonlocal unitary within a single sweep of the itinerant ancilla along the qubits chain) we allow the ancilla to sweep back and forth through the qubit chain and thereby perform multiple bipartite operations at various stages on each qubit. For instance, it can be easily verified that CNOT can be implemented through two rounds of ancilla operations each performed in a sequential manner.

\section{ACKNOWLEDGMENTS}

I gratefully acknowledge stimulating discussions with Enrique Solano, Lucas Lamata, David Pérez-García, Andreas Weichselbaum, Jan von Delft, Jens Eisert, Mehdi Yaghouti, and Farshad Ebrahimi. I particularly would like to thank Andreas Weichselbaum for constructive comments on the manuscript. This work was supported by the Spintronics RTN, the DFG (SFB 631, De-730/3-2), and DFG under SFB 689. Financial support from the German Excellence Initiative via the Nanosystems Initiative Munich (NIM) is gratefully acknowledged. I am also grateful to Universidad del País Vasco for support and hospitality.
[1] M. A. Nielsen and I. L. Chuang, Quantum Computation and Quantum Information (Cambridge University Press, Cambridge, 2000).

[2] C. Schön, E. Solano, F. Verstraete, J. I. Cirac, and M. M. Wolf, Phys. Rev. Lett. 95, 110503 (2005).

[3] C. Schön, K. Hammerer, M. M. Wolf, J. I. Cirac, and E. Solano, Phys. Rev. A 75, 032311 (2007).

[4] H. Saberi, A. Weichselbaum, L. Lamata, D. Pérez-García, J. von Delft, and E. Solano, Phys. Rev. A 80, 022334 (2009).

[5] L. Lamata, J. León, D. Pérez-García, D. Salgado, and E. Solano, Phys. Rev. Lett. 101, 180506 (2008).

[6] K. Kraus, States, Effects and Operations (Springer, Berlin, 1983).

[7] M. A. Nielsen, Ph.D. thesis, University of New Mexico, 1998.

[8] M. A. Nielsen, C. M. Dawson, J. L. Dodd, A. Gilchrist, D. Mortimer, T. J. Osborne, M. J. Bremner, A. W. Harrow, and A. Hines, Phys. Rev. A 67, 052301 (2003).

[9] I. P. McCulloch, J. Stat. Mech. Theory Exp. (2007) P10014.

[10] G. M. Crosswhite and D. Bacon, Phys. Rev. A 78, 012356 (2008).

[11] B. Pirvu, V. Murg, J. I. Cirac, and F. Verstraete, New J. Phys. 12, 025012 (2010).

[12] We remark that the last line of Eq. (4) has also been obtained in Ref. [5], although without making any reference to Kraus representation.

[13] We adopt this convention in the remainder of the paper too since exploiting the orthogonality feature of Schmidt vectors (or operators) alone suffices for the purpose of our arguments.

[14] U. Fano, Rev. Mod. Phys. 55, 855 (1983).

[15] I. Bengtsson and K. Zyczkowski, Geometry of Quantum States: An Introduction to Quantum Entanglement (Cambridge University Press, Cambridge, 2006).

[16] H.-P. Breuer and F. Petruccione, The Theory of Open Quantum Systems (Oxford University Press, Oxford, 2007).

[17] P. Štelmachovič and V. Bužek, Phys. Rev. A 64, 062106 (2001).
[18] A. Peres, Phys. Rev. Lett. 77, 1413 (1996).

[19] W. Dür, J. I. Cirac, and R. Tarrach, Phys. Rev. Lett. 83, 3562 (1999).

[20] M. Lewenstein, D. Bruß, J. I. Cirac, B. Kraus, M. Kus, J. Samsonowicz, A. Sanpera, and R. Tarrach, J. Mod. Opt. 47, 2481 (2000).

[21] O. Gühne and M. Seevinck, New J. Phys. 12, 053002 (2010).

[22] A tripartite system $12 a$ is called 1-qubit biseparable with respect to partition $a$ (ancilla) if it is separable with respect to the ancilla but nonseparable with respect to qubits 12 . Formally, $\rho_{12 a}=$ $\sum_{i}\left|\psi_{\mathrm{i}}\right\rangle_{\mathrm{a}}\left\langle\psi_{\mathrm{i}}|\otimes| \phi_{\mathrm{i}}\right\rangle_{12}\left\langle\phi_{\mathrm{i}}\right|$, where $\left|\psi_{\mathrm{i}}\right\rangle$ are (unnormalized) states of the ancilla and $\left|\phi_{\mathrm{i}}\right\rangle$ are states of qubits 12 [19].

[23] It is easy to show that there exist two-qubit unitary operators with Schmidt numbers 1, 2, and 4, but not 3 [8].

[24] The Frobenius or Hilbert-Schmidt norm of an operator is defined by $O_{F}=\sqrt{\operatorname{Tr}\left(O^{\dagger} O\right)}$.

[25] D. P. Bertsekas, Constrained Optimization and Lagrange Multiplier Methods (Athena Scientific, Belmont, 1996).

[26] F. Verstraete, J. J. García-Ripoll, and J. I. Cirac, Phys. Rev. Lett. 93, 207204 (2004).

[27] F. Verstraete, D. Porras, and J. I. Cirac, Phys. Rev. Lett. 93, 227205 (2004).

[28] H. Saberi, A. Weichselbaum, and J. von Delft, Phys. Rev. B 78, 035124 (2008).

[29] H. Saberi, Ph.D. thesis, Ludwig-Maximilians University, 2009.

[30] A. Weichselbaum, F. Verstraete, U. Schollwöck, J. I. Cirac, and J. von Delft, Phys. Rev. B 80, 165117 (2009).

[31] For $N=3$ any bipartite decomposition of the qubits yields a Schmidt rank identical to that of $N=2$ [35].

[32] P. Zanardi, Phys. Rev. A 63, 040304 (2001).

[33] X. Wang and P. Zanardi, Phys. Rev. A 66, 044303 (2002).

[34] X. Wang, B. C. Sanders, and D. W. Berry, Phys. Rev. A 67, 042323 (2003).

[35] G. Vidal, Phys. Rev. Lett. 91, 147902 (2003). 